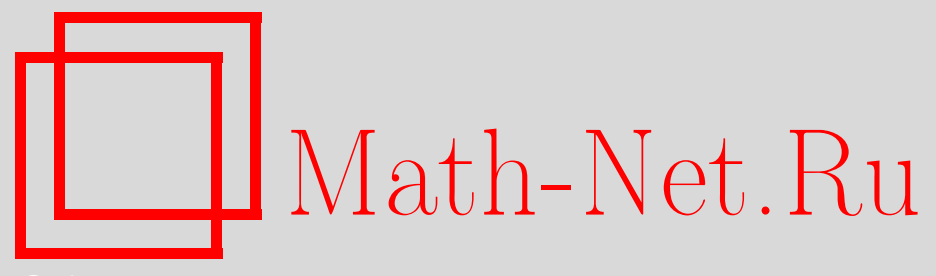

К. Б. Сабитов, Н. В. Мартемьянова, К вопросу о корректности обратных задач для неоднородного уравнения Гельмгольца, Вестн. Сам. гос. техн. ун-та. Сер. Физ.-мат. науки, 2018, номер 2, 269 292

DOI: https://doi.org/10.14498/vsgtu1600

Использование Общероссийского математического портала MathNet.Ru подразумевает, что вы прочитали и согласны с пользовательским соглашением

http://www . mathnet.ru/rus/agreement

Параметры загрузки:

IP: 54.209 .52 .79

26 апреля 2023 г., 17:51:46

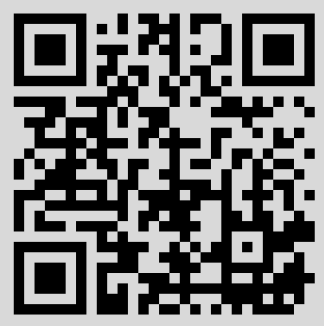


Вестн. Сам. гос. техн. ун-та. Сер. Физ.-мат. науки. 2018. Т. 22, № 2. С. $269-292$ ISSN: 2310-7081 (online), 1991-8615 (print)

УДК 517.956.6

\title{
К вопросу о корректности обратных задач для неоднородного уравнения Гельмгольца
}

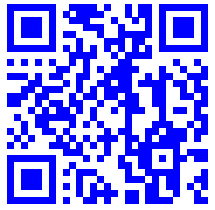

\author{
К. Б. Сабитов ${ }^{1,2}$, Н. В. Мартемъянова \\ 1 Самарский государственный технический университет, \\ Россия, 443100, Самара, ул. Молодогвардейская, 244. \\ 2 Самарский государственный социально-педагогический университет, \\ Россия, 443099, Самара, ул. Горького, 65/67. \\ 3 Самарский национальный исследовательский университет \\ имени академика С.П. Королева, \\ Россия, 443086, Самара, Московское ш., 34.
}

\section{Аннотация}

Для уравнения Гельмгольца в прямоугольной области изучены начально-граничная задача и ее нелокальные модификации, и обратные задачи по его отысканию правой части. Решения прямых задач с нелокальными граничными условиями и обратных задач построены в явном виде как суммы ортогональных рядов по системе собственных функций одномерной спектральной задачи Штурма-Лиувилля. Доказаны соответствующие теоремы единственности решения всех поставленных задач. Установлены достаточные условия на граничные функции, которые гарантируют теоремы существования и устойчивости решения предложенных новых постановок задач.

Ключевые слова: уравнение Гельмгольца, начально-граничная задача, нелокальные задачи, обратные задачи, единственность, существование, ряд, устойчивость, интегральные уравнения.

Получение: 10 января 2018 г. / Исправление: 21 апреля 2018 г. / Принятие: 11 июня 2018 г. / Публикация онлайн: 27 июня 2018 г.

Введение. В связи с изучением обратной задачи по определению правой части уравнения смешанного эллиптико-гиперболического типа, например, известного уравнения Лаврентьева-Бицадзе

$$
u_{x x}+(\operatorname{sgn} y) u_{y y}=\Phi(x, y)
$$

\section{Научная статья}

(2) (1) Контент публикуется на условиях лицензии Creative Commons Attribution 4.0 International (https://creativecommons.org/licenses/by/4.0/deed.ru)

\section{Образец для цитирования}

Сабитов К. Б., Мартемьянова Н. В. К вопросу о корректности обратных задач для неоднородного уравнения Гельмгольца // Вестн. Сам. гос. техн. ун-та. Сер. Физ.мат. науки, 2018. Т. 22, № 2. C. 269-292. doi: 10.14498/vsgtu1600.

\section{Сведения об авторах}

Камиль Басирович Сабитов (1) http://orcid.org/0000-0001-9516-2704

доктор физико-математических наук, профессор; профессор, каф. высшей математики ${ }^{1}$; профессор, каф. физики, математики и методики обучения ${ }^{2}$; e-mail: sabitov_fmf@mail.ru

Нина Викторовна Мартемьянова (1) http://orcid.org/0000-0001-8025-0234

кандидат физико-математических наук; доцент; каф. высшей математики ${ }^{3}$;

e-mail: ninamartem@yandex.ru 
в прямоугольной области $G=\{(x, y) \mid 0<x<l,-\alpha<y<\beta\}$, где

$$
\Phi(x, y)= \begin{cases}\Phi_{1}(x, y)=f_{1}(x) g_{1}(y), & y>0 \\ \Phi_{2}(x, y)=f_{2}(x) g_{2}(y), & y<0\end{cases}
$$

возникает необходимость исследования обратных задач по отысканию правых частей уравнений $u_{x x}+u_{y y}=\Phi_{1}(x, y)$ в $G_{+}=G \cap\{y>0\}$ и $u_{x x}-u_{y y}=$ $=\Phi_{2}(x, y)$ в $G_{-}=G \cap\{y<0\}$. Ранее в наших работах [1-6] были изучены обратные задачи по определению функций $u(x, y)$ и $f_{1}(x)=f_{2}(x)=f(x)$ и $u(x, y), f_{1}(x)$ и $f_{2}(x), f_{1}(x) \neq f_{2}(x)$ для уравнения $(*)$ в области $G$ при $g_{1}(y)=g_{2}(y) \equiv 1$ с локальными и нелокальными граничными условиями. Эти исследования не позволяют пока построить теорию обратных задача для уравнения $(*)$. В связи с этим важно предварительно исследовать обратные задачи для уравнения $(*)$ в областях эллиптичности и гиперболичности.

Отметим, что задачи оптимизации и связанные с ними обратные задачи, например, задача оптимизации сопла Лаваля, являются предметом исследований работ $[7,8]$.

Обратные задачи разного плана для классических уравнений математической физики и для более общих дифференциальных уравнений в частных производных различных типов изучены достаточно полно; усилиями многих математиков создана теория обратных задач (см. [9-16] и приведенную там обширную библиографию).

В данной работе мы исследуем прямые и обратные задачи для уравнений Лапласа и Гельмгольца. К нашим исследованиям близки работы [17-20], где для уравнения Пуассона и более общего уравнения эллиптического типа изучены обратные задачи по отысканию функций $u(x, y)$ и $f_{1}(x)$ в прямоугольной области и областях более общего вида с заданием граничного условия на всей границе области и дополнительной информации о функции $u(x, y)$ внутри области и доказаны теоремы единственности и существования в классах гладких функций.

Отметим также работы [21-23], в которых изучена задача по определению сомножителя правой части операторного уравнения эллиптического типа, где установлен критерий единственности и найдено необходимое и достаточное условие разрешимости поставленной задачи.

В отличие от этих работ, в данной статье решения поставленных обратных задач построены в явном виде как суммы рядов, доказаны соответствующие теоремы единственности, существования и устойчивости.

В связи с этим п. 1 данной работы посвящен изучению начально-граничной задачи, имеющей важное значение в геофизике [13, с. 131], [16, с. 254], и двух ее нелокальных модификаций для уравнения Гельмгольца

$$
u_{x x}+u_{y y}+c u=F(x, y)=f(x) g(y)
$$

в прямоугольной области $G_{+}$, которые являются корректными по Адамару и будут использованы в последующем при постановке обратных задач.

В п. 2 изучены обратные задачи по отысканию правой части уравнения $(* *)$.

В пп. 2.1 рассмотрен случай, когда $g(y) \equiv 1$ и неизвестными являются функции $u(x, y)$ и $f(x)$. Решение построено в виде сумм ортогональных 
рядов и доказаны теоремы единственности, существования и устойчивости решения.

В пп. 2.2 изучен случай, когда $g(y) \not \equiv 1$ и неизвестными также являются функции $u(x, y)$ и $f(x)$. Установлен критерий единственности решения поставленной обратной задачи. Аналогично пп. 2.1, решение построено в виде сумм ортогональных рядов и доказаны теоремы существования и устойчивости решения поставленной обратной задачи.

В пп. 2.3 изучается обратная задача, в которой неизвестными являются функции $u(x, y)$ и $g(y)$. На основании результатов п. 1 относительно функции $g(y)$ получено интегральное уравнение Фредгольма второго рода, из которого при определенных условиях на данные следует однозначная разрешимость обратной задачи.

\section{1. Начально-граничная задача и ее нелокальные модификации.} Рассмотрим неоднородное уравнение Гельмгольца

$$
L u=u_{x x}+u_{y y}+c u=F(x, y),
$$

где $c=$ const $<(\pi / l)^{2}$, в прямоугольной области $D=\{(x, y) \mid 0<x<l$, $0<y<\beta\}$ и следующую начально-граничную задачу.

ЗАДАчА 1. Найти функцию $u(x, y)$, удовлетворяющую условиям

$$
\begin{gathered}
u(x, y) \in C^{2}(D) \cap C^{1}(D \cup\{0 \leqslant x \leqslant l, y=0\}) \cap C(\bar{D}), \\
L u(x, y) \equiv F(x, y), \quad(x, y) \in D, \\
u(0, y)=u(l, y)=0, \quad 0 \leqslant y \leqslant \beta, \\
u(x, 0)=\varphi(x), \quad 0 \leqslant x \leqslant l, \\
u_{y}(x, 0)=\psi(x), \quad 0 \leqslant x \leqslant l,
\end{gathered}
$$

где $F(x, y), \varphi(x), \psi(x)$ - заданные достаточно гладкие функции.

Отметим, что к этой задаче сводятся двумерные задачи Коши для уравнения Лапласа, связанные с изучением стационарных электростатических или магнитных полей с целью поиска полезных ископаемых [13, с. 131], [16, с. 254].

Рассматриваемая задача (2)-(6) некорректна по Адамару. Пусть $F(x, y) \equiv 0$. Тогда решением задачи 1 является функция

$$
u_{k}(x, y)=\frac{1}{k} \sin \mu_{k} x\left(a \operatorname{ch} \lambda_{k} y+\frac{b}{\lambda_{k}} \operatorname{sh} \lambda_{k} y\right)
$$

с начальными функциями

$$
\varphi_{k}(x)=\frac{a}{k} \sin \mu_{k} x, \quad \psi_{k}(x)=\frac{b}{k} \sin \mu_{k} x,
$$

$a, b$ - вещественные постоянные, $k \in \mathbb{N}$.

Из (7) и (8) следует, что с ростом $k$ начальные функции $\varphi_{k}(x)$ и $\psi_{k}(x)$ стремятся к нулю, а $u_{k}(x, y)$ неограниченно растет, т. е. решение задачи 1 неустойчиво.

ТеОрема 1. Если существует решение задачи (2)-(6), удовлетворяющее условиям

$$
\lim _{x \rightarrow 0+0} u_{x} \sin x=\lim _{x \rightarrow l-0} u_{x} \sin \frac{\pi x}{l}=0,
$$


то оно единственно.

До ка з а тель ст в о. Пусть существует решение задачи (2)-(6), удовлетворяющее условиям (9). Следуя [3], введем функции

$$
u_{k}(y)=\frac{2}{l} \int_{0}^{l} u(x, y) X_{k}(x) d x, \quad k \in \mathbb{N},
$$

где $X_{k}(x)=\sin \mu_{k} x, \mu_{k}=\pi k / l-$ собственные функции спектральной задачи

$$
X^{\prime \prime}(x)+\mu^{2} X(x)=0, \quad X(0)=X(l)=0,
$$

которые образуют ортогональную и полную систему в пространстве $L_{2}[0, l]$.

Рассмотрим

$$
u_{k, \varepsilon}(y)=\frac{2}{l} \int_{\varepsilon}^{l-\varepsilon} u(x, y) X_{k}(x) d x
$$

где $\varepsilon>0$ - достаточно малое число. Дифференцируя равенство (11) дважды и используя уравнение (1), имеем

$$
\begin{aligned}
u_{k, \varepsilon}^{\prime \prime}(y)= & \frac{2}{l} \int_{\varepsilon}^{l-\varepsilon} u_{y y}(x, y) X_{k}(x) d x=\frac{2}{l} \int_{\varepsilon}^{l-\varepsilon}\left[F(x, y)-u_{x x}-c u\right] X_{k}(x) d x= \\
& =\frac{2}{l} \int_{\varepsilon}^{l-\varepsilon} F(x, y) X_{k}(x) d x-c u_{k, \varepsilon}-\frac{2}{l} \int_{\varepsilon}^{l-\varepsilon} u_{x x}(x, y) X_{k}(x) d x .
\end{aligned}
$$

В последнем интеграле правой части (12) после интегрирования по частям два раза и перехода к пределу при $\varepsilon \rightarrow 0$ с учетом условий (4)-(9) получим, что функции (10) удовлетворяют дифференциальному уравнению

$$
u_{k}^{\prime \prime}(y)-\lambda_{k}^{2} u_{k}(y)=F_{k}(y) .
$$

Здесь $\lambda_{k}^{2}=\mu_{k}^{2}-c>0$,

$$
F_{k}(y)=\frac{2}{l} \int_{0}^{l} F(x, y) X_{k}(x) d x .
$$

Общее решение уравнения (13) определим в виде

$$
u_{k}(y)=a_{k} e^{\lambda_{k} y}+b_{k} e^{-\lambda_{k} y}-\frac{1}{2 \lambda_{k}} F_{1 k}(y)-\frac{1}{2 \lambda_{k}} F_{2 k}(y),
$$

удобном для дальнейших исследований. Здесь $a_{k}$ и $b_{k}-$ произвольные постоянные,

$$
F_{1 k}(y)=\int_{y}^{\beta} F_{k}(t) e^{-\lambda_{k}(t-y)} d t, \quad F_{2 k}(y)=\int_{0}^{y} F_{k}(t) e^{-\lambda_{k}(y-t)} d t .
$$

Для нахождения $a_{k}$ и $b_{k}$ воспользуемся граничными условиями $(5)$ и (6):

$$
u_{k}(0)=\frac{2}{l} \int_{0}^{l} u(x, 0) \sin \mu_{k} x d x=\frac{2}{l} \int_{0}^{l} \varphi(x) \sin \mu_{k} x d x=\varphi_{k}
$$




$$
u_{k}^{\prime}(0)=\frac{2}{l} \int_{0}^{l} u_{y}(x, 0) \sin \mu_{k} x d x=\frac{2}{l} \int_{0}^{l} \psi(x) \sin \mu_{k} x d x=\psi_{k} .
$$

Теперь, удовлетворяя функции (14) условиям (15) и (16), получим систему

$$
\left\{\begin{array}{l}
a_{k}+b_{k}=\varphi_{k}+\frac{1}{2 \lambda_{k}} F_{1 k}(0), \\
a_{k}-b_{k}=\frac{\psi_{k}}{\lambda_{k}}+\frac{1}{2 \lambda_{k}} F_{1 k}(0),
\end{array}\right.
$$

из которой определяются $a_{k}$ и $b_{k}$ :

$$
a_{k}=\frac{\varphi_{k}+\psi_{k} / \lambda_{k}}{2}+\frac{1}{2 \lambda_{k}} F_{1 k}(0), \quad b_{k}=\frac{\varphi_{k}-\psi_{k} / \lambda_{k}}{2} .
$$

Подставляя найденные значения $a_{k}$ и $b_{k}$ в $(14)$, получим явный вид функций $u_{k}(y)$ :

$$
u_{k}(y)=\varphi_{k} \operatorname{ch} \lambda_{k} y+\frac{\psi_{k}}{\lambda_{k}} \operatorname{sh} \lambda_{k} y+\frac{1}{2 \lambda_{k}} e^{\lambda_{k} y} F_{1 k}(0)-\frac{1}{2 \lambda_{k}} F_{1 k}(y)-\frac{1}{2 \lambda_{k}} F_{2 k}(y) .
$$

Пусть теперь $\varphi(x) \equiv 0, \psi(x) \equiv 0, F(x, y) \equiv 0$. Тогда все $\varphi_{k}=\psi_{k} \equiv 0$, $F_{k}(t) \equiv 0$ и из (17) и (10) следует, что при всех $k \in \mathbb{N}$

$$
\int_{0}^{l} u(x, y) \sin \mu_{k} x d x=0 \text {. }
$$

Из (18) в силу полноты системы $\left\{\sin \mu_{k} x\right\}_{k \geqslant 1}$ в $L_{2}[0, l]$ следует, что $u(x, y)=0$ почти всюду на $[0, l]$ при любом $y \in[0, \beta]$. Поскольку в силу условия (2) функция $u(x, y)$ непрерывна на $\bar{D}$, она тождественно равна нулю на $\bar{D}$.

ЗАмечание 1. Отметим, что в работе [16, с. 254-256] приведено доказательство теоремы единственности решения задачи 1 в классе функций из $C^{4}(\bar{D})$, представимых в виде ряда

$$
u(x, y)=\sum_{k=1}^{\infty} u_{k}(y) \sin \pi k x
$$

где $u_{k}(y) \in C^{4}[0,1], l=\beta=1$. В теореме 1 условия (9) означают, что производная $u_{x}$ вблизи отрезков $x=0$ и $x=l$ может иметь особенности порядка меньше единицы.

Формально решение задачи 1 можно построить в виде суммы ряда

$$
u(x, y)=\sum_{k=1}^{\infty} u_{k}(y) \sin \mu_{k} x
$$

где коэффициенты $u_{k}(y)$ однозначно определены по формуле (17).

Поскольку система $\left\{\sin \mu_{k} x\right\}_{k \geqslant 1}$ образует ортогональный базис в $L_{2}[0, l]$, ряд (19) сходится в $L_{2}[0, l]$ при любом фиксированном $y \in[0, \beta]$. Доказать 
равномерную сходимость этого ряда на $\bar{D}$ не удастся, так как коэффициенты $u_{k}(y)$ при больших $k$ растут по закону экспоненты $e^{\lambda_{k} y}$. Поэтому доказательство существования решения задачи 1 в указанном классе функций (2) и (9) остается под большим вопросом.

Возникает вопрос: можно ли, не меняя структуру решения, поправить постановку задачи (2)-(6) так, чтобы измененная задача стала корректной?

Здесь рассмотрим два варианта. В первом варианте предлагается оставить граничные условия (4) и (5) без изменения, а условие (6) заменить нелокальным граничным условием

$$
u_{y}(x, 0)-u_{y}(x, \beta)=\widetilde{\psi}(x), \quad 0 \leqslant x \leqslant l .
$$

Эту задачу назовем ЗАдАчЕй 2. В этом случае решение задачи 2 ищется в классе функций

$$
u(x, y) \in C^{2}(D) \cap C^{1}(D \cup\{y=0,0 \leqslant x \leqslant l\} \cup\{y=\beta, 0 \leqslant x \leqslant l\}) \cap C(\bar{D}) .
$$

Во втором варианте предлагается в задаче 1 заменить условие (5) на нелокальное условие

$$
u(x, 0)-u(x, \beta)=\widetilde{\varphi}(x), \quad 0 \leqslant x \leqslant l .
$$

Эту задачу назовем ЗАДАчЕй 3 . В условиях $(20)$ и $(21)$ функции $\widetilde{\psi}(x)$ и $\widetilde{\varphi}(x)$ считаются известными и достаточно гладкими.

Решение задачи $\left(2^{\prime}\right),(3)-(5)$ и (20) (задачи 2), аналогично решению задачи 1, можно построить в виде суммы ряда

$$
u(x, y)=\sum_{k=1}^{\infty} u_{k}(y) \sin \mu_{k} x
$$

Действительно, вводя функции (10) относительно $u_{k}(y)$, получаем уравнение (13), общее решение которого определяется по формуле (14). Для определения неизвестных коэффициентов $a_{k}$ и $b_{k}$ воспользуемся граничными условиями (5) и (20), т. е. условиями (15) и

$$
\begin{aligned}
& u_{k}^{\prime}(0)-u_{k}^{\prime}(\beta)=\frac{2}{l} \int_{0}^{l}\left[u_{y}(x, 0)-u_{y}(x, \beta)\right] \sin \mu_{k} x d x= \\
&=\frac{2}{l} \int_{0}^{l} \widetilde{\psi}(x) \sin \mu_{k} x d x=\widetilde{\psi}_{k} .
\end{aligned}
$$

Удовлетворив функции (14) граничным условиям (15) и (23), найдем искомые коэффициенты:

$$
\begin{gathered}
a_{k}=\frac{1}{2\left(\operatorname{ch} \lambda_{k} \beta-1\right)}\left[\varphi_{k}\left(e^{-\lambda_{k} \beta}-1\right)-\frac{\widetilde{\psi}_{k}}{\lambda_{k}}+\frac{F_{1 k}(0)}{2 \lambda_{k}}\left(e^{-\lambda_{k} \beta}-2\right)-\frac{F_{2 k}(\beta)}{2 \lambda_{k}}\right] \\
b_{k}=\frac{1}{2\left(\operatorname{ch} \lambda_{k} \beta-1\right)}\left[\frac{\widetilde{\psi}_{k}}{\lambda_{k}}-\varphi_{k}\left(1-e^{\lambda_{k} \beta}\right)+\frac{e^{\lambda_{k} \beta} F_{1 k}(0)}{2 \lambda_{k}}+\frac{F_{2 k}(\beta)}{2 \lambda_{k}}\right]
\end{gathered}
$$


Подставляя найденные значения $a_{k}$ и $b_{k}$ в формулу $(14)$, получим явный вид искомых функций:

$$
\begin{aligned}
& u_{k}(y)=\varphi_{k} \frac{\operatorname{ch} \lambda_{k}(\beta-y)-\operatorname{ch} \lambda_{k} y}{\left(\operatorname{ch} \lambda_{k} \beta-1\right)}-\widetilde{\psi}_{k} \frac{\operatorname{sh} \lambda_{k} y}{\lambda_{k}\left(\operatorname{ch} \lambda_{k} \beta-1\right)}+ \\
& +\frac{F_{1 k}(0)\left[\operatorname{ch} \lambda_{k}(\beta-y)-\operatorname{ch} \lambda_{k} y-\operatorname{sh} \lambda_{k} y\right]}{2 \lambda_{k}\left(\operatorname{ch} \lambda_{k} \beta-1\right)}- \\
& \quad-\frac{1}{2 \lambda_{k}} F_{1 k}(y)-\frac{F_{2 k}(\beta) \operatorname{sh} \lambda_{k} y}{2 \lambda_{k}\left(\operatorname{ch} \lambda_{k} \beta-1\right)}-\frac{1}{2 \lambda_{k}} F_{2 k}(y) .
\end{aligned}
$$

ЛЕмма 1. При любом $у \in[0, \beta]$ для функиий (24) справедливы оценки

$$
\begin{gathered}
\left|u_{k}(y)\right| \leqslant C_{1}\left(\left|\varphi_{k}\right|+\frac{1}{k}\left|\widetilde{\psi}_{k}\right|+\frac{1}{k^{3 / 2}}\left\|F_{k}(y)\right\|_{L_{2}}\right), \\
\left|u_{k}^{\prime}(y)\right| \leqslant C_{2}\left(k\left|\varphi_{k}\right|+\left|\widetilde{\psi}_{k}\right|+\frac{1}{k^{1 / 2}}\left\|F_{k}(y)\right\|_{L_{2}}\right), \\
\left|u_{k}^{\prime \prime}(y)\right| \leqslant C_{3}\left(k^{2}\left|\varphi_{k}\right|+k\left|\widetilde{\psi}_{k}\right|+k^{1 / 2}\left\|F_{k}(y)\right\|_{L_{2}}\right) .
\end{gathered}
$$

Здесь и ниже $C_{i}$ - положительные постоянные, зависящие, вообще говоря, от данных задачи, $\beta$, с и $l ;\left\|F_{k}(y)\right\|_{L_{2}}=\left\|F_{k}(y)\right\|_{L_{2}[0, \beta]}$.

Доказ ательство. Отметим, что

$$
\begin{gathered}
\frac{\left|\operatorname{ch} \lambda_{k}(\beta-y)-\operatorname{ch} \lambda_{k} y\right|}{\operatorname{ch} \lambda_{k} \beta-1} \leqslant 1, \quad 0 \leqslant \frac{\operatorname{sh} \lambda_{k} y}{\operatorname{ch} \lambda_{k} \beta-1} \leqslant \frac{1+e^{-\lambda_{1} \beta}}{1-e^{-\lambda_{1} \beta}}, \\
0<\frac{\operatorname{sh} \lambda_{k}(\beta-y)+\operatorname{sh} \lambda_{k} y}{\operatorname{ch} \lambda_{k} \beta-1} \leqslant \frac{1+e^{-\lambda_{1} \beta}}{1-e^{-\lambda_{1} \beta}}, \quad 0<\frac{\operatorname{ch} \lambda_{k} y}{\operatorname{ch} \lambda_{k} \beta-1} \leqslant \frac{1+e^{-2 \lambda_{1} \beta}}{\left(1-e^{-\lambda_{1} \beta}\right)^{2}}, \\
\left|F_{1 k}(y)\right| \leqslant \frac{\left\|F_{k}(y)\right\|_{L_{2}}}{\sqrt{2 \lambda_{k}}}, \quad\left|F_{2 k}(y)\right| \leqslant \frac{\left\|F_{k}(y)\right\|_{L_{2}}}{\sqrt{2 \lambda_{k}}} .
\end{gathered}
$$

Тогда оценки (25)-(27) непосредственно следуют из формулы (24).

На основании оценок (25)-(27) нетрудно доказать следующие утверждения.

Teоpema 2. Ecлu $\varphi(x) \in C^{3}[0, l], \varphi(0)=\varphi^{\prime \prime}(0)=0, \widetilde{\psi}(x) \in C^{2}[0, l], \widetilde{\psi}(0)=$ $=\widetilde{\psi}(l)=0 ; F(x, y), F_{x}^{\prime}(x, y), F_{x x}^{\prime \prime}(x, y) \in C(\bar{D}), F(0, y)=F(l, y)=0$ npu $0 \leqslant y \leqslant \beta$, то существует единственное решение $u(x, y)$ задачи $\left(2^{\prime}\right),(3)$ (5) u (20), которое определяется как сумма ряда (22), а коэфффичиенты - по бормуле (24), при этом $u(x, y) \in C^{2}(\bar{D})$.

Доказательство. В силу леммы 1 ряд (22) и ряды, полученные из него почленным дифференцированием до второго порядка включительно, в замкнутой области $\bar{D}$ мажорируются числовым рядом

$$
C_{4} \sum_{k=1}^{+\infty} k^{2}\left|\varphi_{k}\right|+k\left|\widetilde{\psi}_{k}\right|+k^{1 / 2}\left\|F_{k}(y)\right\|_{L_{2}},
$$

который в силу условий теоремы сходится [24, с. 90-94]. Тогда в силу признака Вейерштрасса указанные ряды сходятся равномерно на $\bar{D}$. Поэтому 
сумма ряда $(22)$ принадлежит пространству $C^{2}(\bar{D})$ и удовлетворяет граничным условиям (4), (5) и (20).

Теорема 3. Для решения задачи (2'), (3)-(5) и (20) справедливы оценки устойчивости:

$$
\begin{gathered}
\|u(x, y)\|_{L_{2}[0, l]} \leqslant C_{5}\left(\|\varphi(x)\|_{L_{2}[0, l]}+\|\widetilde{\psi}(x)\|_{L_{2}[0, l]}+\|F(x, y)\|_{L_{2}(D)}\right) \\
\|u(x, y)\|_{C(\bar{D})} \leqslant C_{6}\left(\left\|\varphi^{\prime}(x)\right\|_{C[0, l]}+\|\widetilde{\psi}(x)\|_{C[0, l]}+\|F(x, y)\|_{C(\bar{D})}\right) .
\end{gathered}
$$

Доказательство. В силу оценки (25) на основании формулы имеем

$$
\begin{aligned}
\|u(x, y)\|_{L_{2}[0, l]}^{2}=\frac{l}{2} \sum_{k=1}^{\infty} u_{k}^{2}(y) \leqslant & \frac{3 l C_{1}^{2}}{2} \sum_{k=1}^{\infty}\left(\left|\varphi_{k}\right|^{2}+\left|\widetilde{\psi}_{k}\right|^{2}+\frac{1}{k^{3}}\left\|F_{k}(y)\right\|_{L_{2}}^{2}\right) \leqslant \\
& \leqslant C_{5}^{2}\left(\|\varphi\|_{L_{2}[0, l]}^{2}+\|\widetilde{\psi}\|_{L_{2}[0, l]}^{2}+\|F(x, y)\|_{L_{2}(D)}^{2}\right) .
\end{aligned}
$$

Отсюда следует справедливость оценки (28).

Пусть $(x, y)$ - произвольная точка области $\bar{D}$. Тогда из соотношений $(22)$ и $(25)$ будем иметь

$$
|u(x, y)| \leqslant \sum_{k=1}^{\infty}\left|u_{k}(y)\right| \leqslant C_{1} \sum_{k=1}^{\infty}\left(\left|\varphi_{k}\right|+\frac{1}{k}\left|\widetilde{\psi}_{k}\right|+\frac{1}{k^{3 / 2}}\left\|F_{k}(y)\right\|_{L_{2}}\right) .
$$

Теперь, используя представление $\varphi_{k}=\varphi_{k}^{(1)} / \mu_{k}$, где

$$
\varphi_{k}^{(1)}=\frac{2}{l} \int_{0}^{l} \varphi^{\prime}(x) \cos \mu_{k} x d x
$$

оценку

$$
\left\|F_{k}(y)\right\|_{L_{2}}=\left\|F_{k}(y)\right\|_{L_{2}[0, \beta]} \leqslant \sqrt{\frac{2}{l}}\|F(x, y)\|_{L_{2}(D)} \leqslant \sqrt{2 \beta} \max _{\bar{D}}|F(x, y)|
$$

и неравенство Коши-Буняковского из (30), получаем

$$
\begin{aligned}
&|u(x, y)| \leqslant \widetilde{C}_{1} \sum_{k=1}^{\infty}\left(\frac{1}{k}\left|\varphi_{k}^{(1)}\right|+\frac{1}{k}\left|\widetilde{\psi}_{k}\right|+\frac{\|F(x, y)\|_{C(\bar{D})}}{k^{3 / 2}}\right) \leqslant \\
& \leqslant \widetilde{C}_{1} \sum_{k=1}^{\infty}\left(\frac{1}{k^{2}}\right)^{1 / 2}\left[\left(\sum_{k=1}^{\infty}\left|\varphi_{k}^{(1)}\right|^{2}\right)^{1 / 2}+\left(\sum_{k=1}^{\infty}\left|\widetilde{\psi}_{k}\right|^{2}\right)^{1 / 2}\right]+ \\
& \quad+\widetilde{C}_{1}\|F(x, y)\|_{C(\bar{D})} \sum_{k=1}^{\infty} \frac{1}{k^{3 / 2}}= \\
&=\widetilde{C}_{1} \frac{\pi}{\sqrt{6}}\left(\left\|\varphi^{\prime}(x)\right\|_{L_{2}[0, l]}+\| \widetilde{\psi}_{L_{2}[0, l]}\right)+\widetilde{C}_{1} \widetilde{C}_{2}\|F(x, y)\|_{C(\bar{D})} \leqslant
\end{aligned}
$$




$$
\leqslant \widetilde{C}_{1} \frac{\pi \sqrt{l}}{\sqrt{6}}\left(\left\|\varphi^{\prime}(x)\right\|_{C[0, l]}+\|\widetilde{\psi}\|_{C[0, l]}\right)+\widetilde{C}_{1} \widetilde{C}_{2}\|F(x, y)\|_{C(\bar{D})},
$$

где

$$
\widetilde{C}_{1}=C_{1} \max \{1, l / \sqrt{\pi}, \sqrt{2 \beta}\}, \quad \sum_{k=1}^{\infty} \frac{1}{k^{2}}=\frac{\pi^{2}}{6}, \quad \sum_{k=1}^{\infty} \frac{1}{k^{3 / 2}}=\widetilde{C}_{2}>0 .
$$

Из неравенства (31) непосредственно следует оценка (29).

Аналогично задачам 1 и 2, построим решение задачи (2)-(4), (6) и (21) (задачи 3) как сумму ряда

$$
u(x, y)=\sum_{k=1}^{\infty} u_{k}(y) \sin \mu_{k} x
$$

Здесь $u_{k}(y)$ определяется по формуле

$$
\begin{aligned}
& u_{k}(y)=-\widetilde{\varphi}_{k} \frac{\operatorname{ch} \lambda_{k} y}{\operatorname{ch} \lambda_{k} \beta-1}-\frac{\psi_{k}}{\lambda_{k}} \frac{\operatorname{sh} \lambda_{k}(\beta-y)+\operatorname{sh} \lambda_{k} y}{\operatorname{ch} \lambda_{k} \beta-1}- \\
&-\frac{F_{1 k}(0)}{2 \lambda_{k}} \frac{\operatorname{sh} \lambda_{k}(\beta-y)+\operatorname{sh} \lambda_{k} y+\operatorname{ch} \lambda_{k} y}{\operatorname{ch} \lambda_{k} \beta-1}-\frac{1}{2 \lambda_{k}} F_{1 k}(y)+ \\
& \quad+\frac{F_{2 k}(\beta)}{2 \lambda_{k}} \frac{\operatorname{ch} \lambda_{k} y}{\operatorname{ch} \lambda_{k} \beta-1}-\frac{1}{2 \lambda_{k}} F_{2 k}(y),
\end{aligned}
$$

где

$$
\widetilde{\varphi}_{k}=\frac{2}{l} \int_{0}^{l} \widetilde{\varphi}(x) \sin \mu_{k} x d x .
$$

ЛЕмма 2. При любом $у \in[0, \beta]$ справедливы оценки

$$
\begin{gathered}
\left|u_{k}(y)\right| \leqslant C_{7}\left(\left|\widetilde{\varphi}_{k}\right|+\frac{\left|\psi_{k}\right|}{k}+\frac{1}{k^{3 / 2}}\left\|F_{k}(y)\right\|_{L_{2}[0, \beta]}\right), \\
\left|u_{k}^{\prime}(y)\right| \leqslant C_{8}\left(k\left|\widetilde{\varphi}_{k}\right|+\left|\psi_{k}\right|+\frac{1}{k^{1 / 2}}\left\|F_{k}(y)\right\|_{L_{2}[0, \beta]}\right), \\
\left|u_{k}^{\prime \prime}(y)\right| \leqslant C_{9}\left(k^{2}\left|\widetilde{\varphi}_{k}\right|+k\left|\psi_{k}\right|+k^{1 / 2}\left\|F_{k}(y)\right\|_{L_{2}[0, \beta]}\right) .
\end{gathered}
$$

Доказ ательств во аналогично обоснованию леммы 1.

Теорема 4. Если функиия $F(x, y)$ удовлетворяет условиям теоремы 2, $\widetilde{\varphi}(x) \in C^{3}[0, l], \widetilde{\varphi}(0)=\widetilde{\varphi}(l)=\widetilde{\varphi}^{\prime \prime}(0)=\widetilde{\varphi}^{\prime \prime}(l)=0, \psi(x) \in C^{2}[0, l], \psi(0)=\psi(l)=0$, то существует единственное решение и $(x, y)$ задачи (2)-(4), (6) и (21), и это решение определяется рядом (32), у которого коэфбфичиенты находятся по формуле (33), при этом $и(x, y) \in C^{2}(\bar{D})$.

Доказательство следует из установленных в лемме 2 оценок.

Для этой задачи имеют место оценки устойчивости, аналогичные оценкам (28) и (29). 
2. Обратные задачи по отысканию правой части. Здесь для уравнения (1) исследуем обратные задачи по определению правой части, т.е. функции $F(x, y)=f(x) g(y)$.

2.1. В начале рассмотрим случай, когда $g(y) \equiv 1$.

ЗАдАчА 4 . Найти функции $u(x, y)$ и $f(x)$, удовлетворяющие условиям $(2)-$ (6) и, кроме того,

$$
\begin{gathered}
u(x, \beta)=h(x), \quad 0 \leqslant x \leqslant l, \\
f(x) \in C(0, l) \cap L_{2}(0, l),
\end{gathered}
$$

где $\varphi(x), \psi(x)$ и $h(x)$ - заданные достаточно гладкие функции, условие (6) здесь является дополнительным для определения функции $f(x)$.

Теорема 5. Если существует решение задачи 4, удовлетворяющее условиям (9), то оно единственно.

До ка з а тель с т в о. Аналогично доказательству теоремы 1, введем функции (10), которые удовлетворяют уравнению

$$
u_{k}^{\prime \prime}(y)-\lambda_{k}^{2} u_{k}(y)=f_{k},
$$

где

$$
f_{k}=\frac{2}{l} \int_{0}^{l} f(x) \sin \mu_{k} x d x .
$$

Общее решение уравнения (36) определяется по формуле

$$
u_{k}(y)=a_{k} e^{\lambda_{k} y}+b_{k} e^{-\lambda_{k} y}-\frac{f_{k}}{\lambda_{k}^{2}},
$$

где $a_{k}, b_{k}$ и $f_{k}$ - неизвестные постоянные. Для их нахождения воспользуемся граничными условиями (15), (16) и

$$
u_{k}(\beta)=\frac{2}{l} \int_{0}^{l} u(x, \beta) \sin \mu_{k} x d x=\frac{2}{l} \int_{0}^{l} h(x) \sin \mu_{k} x d x=h_{k} .
$$

Удовлетворяя функции (38) граничным условиям (15), (16) и (39), получим систему

$$
\left\{\begin{array}{l}
a_{k}+b_{k}-\frac{f_{k}}{\lambda_{k}^{2}}=\varphi_{k} \\
a_{k}-b_{k}=\frac{\psi_{k}}{\lambda_{k}} \\
a_{k} e^{\lambda_{k} \beta}+b_{k} e^{-\lambda_{k} \beta}-\frac{f_{k}}{\lambda_{k}^{2}}=h_{k}
\end{array}\right.
$$

единственное решение которой определяется формулами

$$
\begin{aligned}
a_{k} & =\frac{h_{k}-\varphi_{k}}{2\left(\operatorname{ch} \lambda_{k} \beta-1\right)}-\frac{\psi_{k}\left(1-e^{-\lambda_{k} \beta}\right)}{2 \lambda_{k}\left(\operatorname{ch} \lambda_{k} \beta-1\right)}, \\
b_{k} & =\frac{\psi_{k}\left(1-e^{\lambda_{k} \beta}\right)}{2 \lambda_{k}\left(\operatorname{ch} \lambda_{k} \beta-1\right)}-\frac{\varphi_{k}-h_{k}}{2\left(\operatorname{ch} \lambda_{k} \beta-1\right)},
\end{aligned}
$$




$$
\frac{f_{k}}{\lambda_{k}^{2}}=\frac{h_{k}}{\operatorname{ch~} \lambda_{k} \beta-1}-\frac{\varphi_{k} \operatorname{ch} \lambda_{k} \beta}{\operatorname{ch} \lambda_{k} \beta-1}-\frac{\psi_{k} \operatorname{sh} \lambda_{k} \beta}{\lambda_{k}\left(\operatorname{ch} \lambda_{k} \beta-1\right)} .
$$

Теперь, подставляя (40)-(42) в (38), найдем функции $u_{k}(y)$ в замкнутом виде:

$$
\begin{aligned}
u_{k}(y)=\varphi_{k} \frac{\operatorname{ch} \lambda_{k} \beta-\operatorname{ch} \lambda_{k} y}{\operatorname{ch} \lambda_{k} \beta-1}+h_{k} \frac{\operatorname{ch} \lambda_{k} y-1}{\operatorname{ch} \lambda_{k} \beta-1}+ & \\
& +\psi_{k} \frac{\operatorname{sh} \lambda_{k} \beta-\operatorname{sh} \lambda_{k} y-\operatorname{sh} \lambda_{k}(\beta-y)}{\lambda_{k}\left(\operatorname{ch} \lambda_{k} \beta-1\right)} .
\end{aligned}
$$

Пусть теперь $\varphi(x)=h(x)=\psi(x) \equiv 0$. Тогда из (42), (43), (10) и (37) при всех $k \in \mathbb{N}$ получим

$$
\int_{0}^{l} u(x, y) \sin \mu_{k} x d x=0, \quad \int_{0}^{l} f(x) \sin \mu_{k} x d x=0 .
$$

В силу полноты системы $\left\{\sin \mu_{k} x\right\}_{k \geqslant 1}$ из равенств (44) следует, что $u(x, y)=0$ почти всюду на $[0, l]$ при любом $y \in[0, \beta]$ и $f(x)=0$ почти всюду на $[0, l]$. В силу условий $(2)$ и $(35)$ функции $u(x, y) \equiv 0$ в $\bar{D}$ и $f(x) \equiv 0$ на $[0, l]$.

Решение задачи 4 определяется как сумма рядов

$$
\begin{gathered}
u(x, y)=\sum_{k=1}^{\infty} u_{k}(y) \sin \mu_{k} x, \\
f(x)=\sum_{k=1}^{\infty} f_{k} \sin \mu_{k} x .
\end{gathered}
$$

Здесь коэффициенты $u_{k}(y)$ и $f_{k}$ находятся соответственно по формулам (43) и $(42)$.

Лемма 3. Для коэффициентов рядов (45) и (46) справедливы оценки

$$
\begin{gathered}
\left|u_{k}(y)\right| \leqslant C_{10}\left(\left|\varphi_{k}\right|+\left|h_{k}\right|+\frac{\left|\psi_{k}\right|}{k}\right), \\
\left|u_{k}^{\prime}(y)\right| \leqslant C_{11}\left(k\left|\varphi_{k}\right|+k\left|h_{k}\right|+\left|\psi_{k}\right|\right), \\
\left|u_{k}^{\prime \prime}(y)\right| \leqslant C_{12}\left(k^{2}\left|\varphi_{k}\right|+k^{2}\left|h_{k}\right|+k\left|\psi_{k}\right|\right), \\
\left|f_{k}\right| \leqslant C_{13}\left(k^{2}\left|\varphi_{k}\right|+\left|h_{k}\right|+k\left|\psi_{k}\right|\right) .
\end{gathered}
$$

Справедливость оценок (47)-(50) непосредственно следует из формул (43) и (42). Исходя из оценок (47)-(50) легко доказывается следующая

Tеорема 6. Ecлu $\varphi(x), h(x) \in C^{3}[0, l], \varphi(0)=\varphi^{\prime \prime}(0)=h(0)=h^{\prime \prime}(0)=0$, $\psi(x) \in C^{2}[0, l], \psi(0)=\psi(l)=0$, то существует единственное решение $u(x, y)$ и $f(x)$ задачи 4 и оно определяется рядами (45) и (46), при этом $u(x, y) \in C^{2}(\bar{D}) u f(x) \in C[0, l]$.

Теорема 7. Для решения задачи 4 справедливы оченки устойчивости:

$$
\|u(x, y)\|_{L_{2}[0, l]} \leqslant C_{14}\left(\|\varphi(x)\|_{L_{2}[0, l]}+\|h(x)\|_{L_{2}[0, l]}+\|\psi(x)\|_{L_{2}[0, l]}\right),
$$




$$
\begin{gathered}
\|f(x)\|_{L_{2}[0, l]} \leqslant C_{15}\left(\left\|\varphi^{\prime \prime}(x)\right\|_{L_{2}[0, l]}+\|h(x)\|_{L_{2}[0, l]}+\left\|\psi^{\prime}(x)\right\|_{L_{2}[0, l]}\right) \\
\|u(x, y)\|_{C(\bar{D})} \leqslant C_{16}\left(\left\|\varphi^{\prime}(x)\right\|_{C[0, l]}+\left\|h^{\prime}(x)\right\|_{C[0, l]}+\|\psi(x)\|_{C[0, l]}\right) \\
\|f(x)\|_{C[0, l]} \leqslant C_{17}\left(\left\|\varphi^{\prime \prime \prime}(x)\right\|_{C[0, l]}+\left\|h^{\prime}(x)\right\|_{C[0, l]}+\left\|\psi^{\prime \prime}(x)\right\|_{C[0, l]}\right) .
\end{gathered}
$$

До каз а тель ст в о оценок (51)-(54) проводится аналогично теореме 3 с использованием оценок (47) и (50).

ЗАмечАниЕ 2. Отметим, что в работе [21] изучена обратная задача для операторного уравнения второго порядка, из которой можно получить явную формулу решения задачи 4, т.е. ряды (45) и (46), которые будут сходиться B $L_{2}[0, l]$.

2.2. Пусть $g(y) \not \equiv 1-$ произвольная непрерывная на $[0, \beta]$ функция. В этом случае аналогично п. 2.1 можно показать единственность решения задачи 4, но при обосновании существования решения возникают принципиальные трудности в силу роста коэффициентов ряда по закону $e^{\lambda_{k} \beta}$. Поэтому здесь предлагается обратная задача на основании прямой задачи 2 или 3. Остановимся на задаче 2.

ЗАДАчА 5 . Найти функции $u(x, y)$ и $f(x)$, удовлетворяющие условиям $\left(2^{\prime}\right)$, $(3)-(5),(20),(34)$ и $(35)$. Здесь $\varphi(x), h(x), \widetilde{\psi}(x)$ и $g(y)$ - заданные достаточно гладкие функции.

Пусть существует решение задачи 5, удовлетворяющее условиям (9). Введем функции (10), которые удовлетворяют уравнению (13):

$$
u_{k}^{\prime \prime}(y)-\lambda_{k}^{2} u_{k}(y)=F_{k}(y)
$$

где

$$
F_{k}(y)=\frac{2}{l} \int_{0}^{l} F(x, y) \sin \mu_{k} x d x=g(y) f_{k} .
$$

В этом случае общее решение уравнения (13) исходя из формулы (14) определяется по формуле

$$
u_{k}(y)=a_{k} e^{\lambda_{k} y}+b_{k} e^{-\lambda_{k} y}-\frac{f_{k}}{2 \lambda_{k}}\left(g_{1 k}(y)+g_{2 k}(y)\right),
$$

где $a_{k}, b_{k}$ и $f_{k}$ - неизвестные постоянные,

$$
g_{1 k}(y)=\int_{y}^{\beta} g(t) e^{-\lambda_{k}(t-y)} d t, \quad g_{2 k}(y)=\int_{0}^{y} g(t) e^{-\lambda_{k}(y-t)} d t
$$

Для определения этих коэффициентов воспользуемся условиями (15), (24) и (39). Удовлетворяя функции (55) граничным условиям (15), (24) и (39), найдем систему

$$
\left\{\begin{array}{l}
a_{k}+b_{k}-\frac{f_{k}}{2 \lambda_{k}} g_{1 k}(0)=\varphi_{k} \\
a_{k} e^{\lambda_{k} \beta}+b_{k} e^{-\lambda_{k} \beta}-\frac{f_{k}}{2 \lambda_{k}} g_{2 k}(\beta)=h_{k} \\
a_{k}\left(1-e^{\lambda_{k} \beta}\right)+b_{k}\left(e^{-\lambda_{k} \beta}-1\right)-\frac{f_{k}}{2 \lambda_{k}}\left[g_{1 k}(0)+g_{2 k}(\beta)\right]=\frac{\widetilde{\psi}_{k}}{\lambda_{k}} .
\end{array}\right.
$$


При условии, что при любом $k \in \mathbb{N}$

$$
\Delta(k)=g_{1 k}(0)+g_{2 k}(\beta)=\int_{0}^{\beta} g(t)\left[e^{-\lambda_{k} t}+e^{-\lambda_{k}(\beta-t)}\right] d t \neq 0
$$

система (57) имеет единственное решение:

$$
\begin{gathered}
a_{k}=\frac{h_{k}-\varphi_{k}-\widetilde{\psi}_{k} / \lambda_{k}}{2\left(e^{\lambda_{k} \beta}-1\right)}+ \\
+\frac{\left(\varphi_{k}+h_{k}\right)\left(1-e^{-\lambda_{k} \beta}\right)+\left(\widetilde{\psi}_{k} / \lambda_{k}\right)\left(1+e^{-\lambda_{k} \beta}\right)}{2\left(e^{\lambda_{k} \beta}-1\right)} \frac{g_{1 k}(0)}{g_{1 k}(0)+g_{2 k}(\beta)}, \\
\frac{f_{k}}{2 \lambda_{k}}=-\frac{\left(\varphi_{k}+h_{k}\right)\left(1-e^{-\lambda_{k} \beta}\right)+\left(\widetilde{\psi}_{k} / \lambda_{k}\right)\left(1+e^{-\lambda_{k} \beta}\right)}{2\left(g_{1 k}(0)+g_{2 k}\right)(\beta)}, \\
b_{k}=\frac{1}{2}\left(\varphi_{k}+h_{k}-\frac{\widetilde{\psi}_{k}}{\lambda_{k}}\right)+\frac{e^{\lambda_{k} \beta}\left(\varphi_{k}-h_{k}+\widetilde{\psi}_{k} / \lambda_{k}\right)}{2\left(e^{\lambda_{k} \beta}-1\right)}- \\
-\frac{e^{\lambda_{k} \beta}\left[\left(\varphi_{k}+h_{k}\right)\left(1-e^{-\lambda_{k} \beta}\right)+\left(\widetilde{\psi}_{k} / \lambda_{k}\right)\left(1+e^{-\lambda_{k} \beta}\right)\right]}{2\left(e^{\lambda_{k} \beta}-1\right)} \frac{g_{1 k}(0)}{g_{1 k}(0)+g_{2 k}(\beta)} .
\end{gathered}
$$

Найденные значения коэффициентов по формулам (59)-(61) подставим в (55). Тогда получим

$$
\begin{aligned}
& u_{k}(y)=\varphi_{k}\left[\frac{e^{\lambda_{k}(\beta-y)}-\operatorname{ch} \lambda_{k} y}{e^{\lambda_{k} \beta}-1}+\frac{\left(1-e^{-\lambda_{k} \beta}\right) g_{1 k}(0)\left(e^{\lambda_{k} y}-e^{\lambda_{k}(\beta-y)}\right)}{2\left(e^{\lambda_{k} \beta}-1\right)\left(g_{1 k}(0)+g_{2 k}(\beta)\right)}+\right. \\
&\left.+\frac{\left(1-e^{-\lambda_{k} \beta}\right)\left(g_{1 k}(y)+g_{2 k}(y)\right)}{2\left(g_{1 k}(0)+g_{2 k}(\beta)\right)}\right]+ \\
&+\left.+\frac{\left(1-e^{-\lambda_{k} \beta}\right)\left(g_{1 k}(y)+g_{2 k}(y)\right)}{2\left(g_{1 k}(0)+g_{2 k}(\beta)\right)}\right]+ \\
&+ \frac{h_{k}\left[\frac{\operatorname{sh} \lambda_{k} y}{e^{\lambda_{k} \beta}-1}+\frac{\left(1-e^{-\lambda_{k} \beta}\right) g_{1 k}(0)\left(e^{\lambda_{k} y}-e^{\lambda_{k}(\beta-y)}\right)}{2\left(e^{\lambda_{k} \beta}-1\right)\left(g_{1 k}(0)+g_{2 k}(\beta)\right)}+\right.}{\lambda_{k}}\left[-\frac{\operatorname{sh} \lambda_{k} y}{e^{\lambda_{k} \beta}-1}+\frac{\left(1+e^{-\lambda_{k} \beta}\right) g_{1 k}(0)\left(e^{\lambda_{k} y}-e^{\lambda_{k}(\beta-y)}\right)}{2\left(e^{\lambda_{k} \beta}-1\right)\left(g_{1 k}(0)+g_{2 k}(\beta)\right)}+\right. \\
&\left.+\frac{\left(1+e^{-\lambda_{k} \beta}\right)\left(g_{1 k}(y)+g_{2 k}(y)\right)}{2\left(g_{1 k}(0)+g_{2 k}(\beta)\right)}\right] .
\end{aligned}
$$

Теперь докажем единственность решения задачи 5 . Пусть $\varphi(x)=h(x)=$ $=\widetilde{\psi}(x) \equiv 0$ и выполнены условия (58) при всех $k \in \mathbb{N}$. Тогда из (60) и (62) следует, что при всех $k \in \mathbb{N}$ функции $u_{k}(y) \equiv 0$ и $f_{k} \equiv 0$. Получим равенства $(44)$, из которых уже следует, что $u(x, y) \equiv 0$ в $\bar{D}$ и $f(x) \equiv 0$ на $[0, l]$.

Пусть при некотором $k=p \in \mathbb{N}$ нарушается условие $(58)$, т.е. $\Delta(p)=0$. Тогда однородная задача 5 (где $\varphi(x)=h(x)=\widetilde{\psi}(x) \equiv 0)$ имеет ненулевое решение:

$$
u_{p}(x, y)=\widetilde{u}_{p}(y) \sin \mu_{p} x
$$




$$
f_{p}(x)=f_{p} \sin \mu_{p} x
$$

где

$$
\widetilde{u}_{p}(y)=\frac{f_{p} g_{1 p}(0)\left(\operatorname{ch} \lambda_{p}(\beta-y)-\operatorname{ch} \lambda_{p} y\right)}{2 \lambda_{p}\left(\operatorname{ch} \lambda_{p} \beta-1\right)}-\frac{f_{p}}{2 \lambda_{p}}\left(g_{1 p}(y)+g_{2 p}(y)\right),
$$

$f_{p} \neq 0-$ произвольная постоянная.

Теперь возникает вопрос о существовании нулей выражения $\Delta(p)$. Из (58) видно, что если функция $g(t)$ на $[0, \beta]$ не меняет знак, т.е. знакопостоянная, то $\Delta(k) \neq 0$ при любом $k \in \mathbb{N}$. Если $g(t)$ меняет знак, т.е. имеет нули на $[0, \beta]$, то уравнение $\Delta(p)=0$ может иметь корни. В качестве примера возьмем функцию

$$
g(t)=\sin (a t+b), \quad a, b \in \mathbb{R}, \quad a \neq 0 .
$$

Тогда выражение $\Delta(p)$ примет вид

$$
\Delta(p)=\frac{2 \sqrt{A_{p}^{2}+B_{p}^{2}}}{\lambda_{p}^{2}+a^{2}} \sin \left(\frac{a \beta}{2}+b\right) \sin \left(\varphi_{p}-\frac{a \beta}{2}\right) .
$$

Здесь

$$
A_{p}=\lambda_{p}\left(1-e^{-\lambda_{p} \beta}\right), \quad B_{p}=a\left(1+e^{-\lambda_{p} \beta}\right), \quad \varphi_{p}=\arcsin \frac{A_{p}}{\sqrt{A_{p}^{2}+B_{p}^{2}}} .
$$

Отсюда следует, что уравнение $\Delta(p)=0$ имеет счетное множество корней относительно $a \beta / 2$.

Таким образом, нами установлен следующий критерий единственности решения задачи 5 .

Теорема 8. Если существует решение задачи 5, удовлетворяющее условиям (9), то оно единственно только тогда, когда выполнены условия (58) при всех $k \in \mathbb{N}$.

При условии (58) решение задачи 5 формально можно построить в виде сумм рядов (45) и (46), где коэффициенты уже находятся соответственно по формулам (62) и (60). Покажем, что суммы этих рядов принадлежат соответственно классам $C^{2}(\bar{D})$ и $C[0, l]$.

Лемма 4. Если функиия $g(y)$ непрерьвна на $[0, \beta] u|g(y)| \geqslant m=$ const $>0$, то существует постоянная $C_{0}>0$ такая, что при всех $k \in \mathbb{N}$

$$
|\Delta(k)| \geqslant \frac{C_{0}}{k}
$$

Д оказ ат ель ст в о. На основании теоремы о среднем выражение $\Delta(k)$ из (58) представим в виде

$$
\Delta(k)=\int_{0}^{\beta} g(t)\left[e^{-\lambda_{k} t}+e^{-\lambda_{k}(\beta-t)}\right] d t=g(\xi) \frac{2\left(1-e^{-\lambda_{k} \beta}\right)}{\lambda_{k}}, \quad 0<\xi<\beta .
$$

Отсюда следует

$$
|\Delta(k)| \geqslant \frac{2 \operatorname{lm}\left(1-e^{-\lambda_{1} \beta}\right)}{\pi k \sqrt{1-c(l /(\pi k))^{2}}}>\frac{C_{0}}{k}
$$


TaK KaK

$$
\sqrt{1-c(l /(\pi k))^{2}} \leqslant \begin{cases}\sqrt{1-c(l / \pi)^{2}} & \text { при } c \leqslant 0, \\ 1 & \text { при } 0<c<(\pi / l)^{2}\end{cases}
$$

где постоянная $C_{0}$ зависит от $l, \beta, c$ и $m$.

Лемма 5. Для коэфбиииентов рядов $u_{k}(y)$ u $f_{k}$ справедливы следующие оц,нки:

$$
\begin{gathered}
\left|u_{k}(y)\right| \leqslant C_{18}\left(\left|\varphi_{k}\right|+\left|h_{k}\right|+\frac{1}{k}\left|\psi_{k}\right|\right), \\
\left|u_{k}^{\prime}(y)\right| \leqslant C_{19}\left(k\left|\varphi_{k}\right|+k\left|h_{k}\right|+\left|\psi_{k}\right|\right), \\
\left|u_{k}^{\prime \prime}(y)\right| \leqslant C_{20}\left(k^{2}\left|\varphi_{k}\right|+k^{2}\left|h_{k}\right|+k\left|\psi_{k}\right|\right), \\
\left|f_{k}\right| \leqslant C_{21}\left(k^{2}\left|\varphi_{k}\right|+k^{2}\left|h_{k}\right|+k\left|\psi_{k}\right|\right) .
\end{gathered}
$$

Доказательств о. Предварительно оценим следующие выражения:

$$
\begin{gathered}
1 \geqslant \frac{e^{\lambda_{k}(\beta-y)}-\operatorname{ch} \lambda_{k} y}{e^{\lambda_{k} \beta}-1} \geqslant-\frac{\operatorname{ch} \lambda_{k} \beta-1}{e^{\lambda_{k} \beta}-1}>-\frac{1}{2}, \\
-1 \leqslant \frac{e^{\lambda_{k} y}-e^{\lambda_{k}(\beta-y)}}{e^{\lambda_{k} \beta}-1} \leqslant 1 \\
\left|g_{1 k}(y)\right| \leqslant \frac{\widetilde{C}_{1}}{k}, \quad\left|g_{2 k}(y)\right| \leqslant \frac{\widetilde{C}_{2}}{k}
\end{gathered}
$$

где $\widetilde{C}_{1}$ и $\widetilde{C}_{2}$ - положительные постоянные, зависящие от $\beta, c, l$ и $\max _{0 \leqslant y \leqslant \beta}|g(y)|$.

Тогда на основании оценки (66) из формул (62) и (60) следует справедливость приведенных оценок (67)-(70).

Теперь на основании оценок (67)-(70) нетрудно обосновать справедливость следующих утверждений.

ТЕОрема 9. Пусть функиии $\varphi(x)$ и $h(x)$ удовлетворяют условиям теоремы $6, \widetilde{\psi}(x) \in C^{2}[0, l], \widetilde{\psi}(0)=\widetilde{\psi}(l)=0$, а функиия $g(y)$ удовлетворяет условиям леммы 4. Тогда существует единственное решение $u(x, y)$ u $f(x)$ задачи 5, которое определяется рядами (45) и (46), при этом коэббичиенты этих рядов находятся по бормулам (62) и $(60) u u(x, y) \in C^{2}(\bar{D})$, $f(x) \in C[0, l]$.

Теорема 10. Для решения задачи 5 имеют место оценки устойчивости (51)-(54), но с другими постоянными, и в этих оценках функцию $\psi(x)$ следует заменить на $\widetilde{\psi}(x)$.

2.3. Пусть теперь $f(x)$ - известная, а $g(y)$ - неизвестная функции. В этом случае дополнительное условие следует уже задать на отрезке $x=x_{0} \in(0, l)$, $0 \leqslant y \leqslant \beta$, а другие краевые условия можно задать, комбинируя граничные условия задач $1-3$ :

$$
\begin{gathered}
u(x, 0)=\varphi(x), \quad 0 \leqslant x \leqslant l, \\
u_{y}(x, 0)-u_{y}(x, \beta)=\widetilde{\psi}(x), \quad 0 \leqslant x \leqslant l ;
\end{gathered}
$$




$$
\begin{gathered}
u_{y}(x, 0)=\psi(x), \quad 0 \leqslant x \leqslant l, \\
u(x, 0)-u(x, \beta)=\widetilde{\varphi}(x), \quad 0 \leqslant x \leqslant l ; \\
u(x, \beta)=h(x), \quad 0 \leqslant x \leqslant l, \\
u_{y}(x, 0)-u_{y}(x, \beta)=\widetilde{\psi}(x), \quad 0 \leqslant x \leqslant l ; \\
u_{y}(x, \beta)=\widetilde{h}(x), \quad 0 \leqslant x \leqslant l, \\
u(x, 0)-u(x, \beta)=\widetilde{\varphi}(x), \quad 0 \leqslant x \leqslant l .
\end{gathered}
$$

Здесь остановимся на первом варианте (условие $\left(B_{1}\right)$ ), т.е. поставим следующую задачу.

ЗАДАчА 6 . Найти функции $u(x, y)$ и $g(y)$, удовлетворяющие условиям $\left(2^{\prime}\right)$, (3)-(5), (20) и, кроме того, условиям

$$
\begin{gathered}
u\left(x_{0}, y\right)=d(y), \quad 0 \leqslant y \leqslant \beta, \quad 0<x_{0}<l, \\
g(y) \in C[0, \beta] .
\end{gathered}
$$

Здесь $\varphi(x), \widetilde{\psi}(x), f(x)$ и $d(y)$ - заданные достаточно гладкие функции, при этом $d(0)=\varphi\left(x_{0}\right)$.

Решение задачи $\left(2^{\prime}\right),(3)-(5),(20)$, т.е. задачи 2 , нами уже построено в виде суммы ряда (22), где коэффициенты $u_{k}(y)$ определяются по формулам (24). Поскольку $F(x, y)=f(x) g(y)$, имеем $F_{k}(y)=f_{k} g(y)$, следовательно,

$$
F_{1 k}(y)=f_{k} g_{1 k}(y), \quad F_{2 k}(y)=f_{k} g_{2 k}(y),
$$

где $g_{1 k}(y)$ и $g_{2 k}(y)$ определены формулами (56). С учетом этих изменений формула (24) принимает вид

$$
\begin{aligned}
& u_{k}(y)=\varphi_{k} \frac{\operatorname{ch} \lambda_{k}(\beta-y)-\operatorname{ch} \lambda_{k} y}{\left(\operatorname{ch} \lambda_{k} \beta-1\right)}-\widetilde{\psi}_{k} \frac{\operatorname{sh} \lambda_{k} y}{\lambda_{k}\left(\operatorname{ch} \lambda_{k} \beta-1\right)}+ \\
&+ \frac{f_{k}}{2 \lambda_{k}}\left[\frac{g_{1 k}(0)\left(\operatorname{ch} \lambda_{k}(\beta-y)-\operatorname{ch} \lambda_{k} y-\operatorname{sh} \lambda_{k} y\right)}{\left(\operatorname{ch} \lambda_{k} \beta-1\right)}-\right. \\
&\left.-g_{1 k}(y)-\frac{g_{2 k}(\beta) \operatorname{sh} \lambda_{k} y}{\operatorname{ch} \lambda_{k} \beta-1}-g_{2 k}(y)\right]= \\
&=\frac{f_{k}}{2 \lambda_{k}}\left[g_{1 k}(0) A_{k}(y)-g_{2 k}(\beta) B_{k}(y)-g_{1 k}(y)-g_{2 k}(y)\right]+C_{k}(y),
\end{aligned}
$$

где

$$
\begin{gathered}
A_{k}(y)=\frac{\operatorname{ch} \lambda_{k}(\beta-y)-\operatorname{ch} \lambda_{k} y-\operatorname{sh} \lambda_{k} y}{\operatorname{ch} \lambda_{k} \beta-1}, \quad B_{k}(y)=\frac{\operatorname{sh} \lambda_{k} y}{\operatorname{ch} \lambda_{k} \beta-1}, \\
C_{k}(y)=\varphi_{k} \frac{\operatorname{ch~} \lambda_{k}(\beta-y)-\operatorname{ch} \lambda_{k} y}{\operatorname{ch} \lambda_{k} \beta-1}-\widetilde{\psi}_{k} \frac{\operatorname{sh} \lambda_{k} y}{\lambda_{k}\left(\operatorname{ch} \lambda_{k} \beta-1\right)} .
\end{gathered}
$$

Теперь удовлетворим ряд (22) граничному условию (71):

$$
u\left(x_{0}, y\right)=\sum_{k=1}^{\infty} u_{k}(y) \sin \mu_{k} x_{0}=d(y), 0 \leqslant y \leqslant \beta,
$$


здесь $u_{k}(y)$ находится по формуле (72).

Предварительно первое слагаемое в квадратных скобках в правой части формулы (72) преобразуем к виду

$$
\begin{aligned}
\widetilde{u}_{k}(y)=g_{1 k}(0) A_{k}(y)-g_{2 k}(\beta) B_{k}(y)-g_{1 k}(y)-g_{2 k}(y)= \\
=A_{k}(y) \int_{0}^{\beta} g(t) e^{-\lambda_{k} t} d t-B_{k}(y) \int_{0}^{\beta} g(t) e^{-\lambda_{k}(\beta-t)} d t- \\
\quad-\int_{y}^{\beta} g(t) e^{-\lambda_{k}(t-y)} d t-\int_{0}^{y} g(t) e^{-\lambda_{k}(y-t)} d t= \\
=\int_{0}^{y} g(t)\left[A_{k}(y) e^{-\lambda_{k} t}-B_{k}(y) e^{-\lambda_{k}(\beta-t)}-e^{-\lambda_{k}(y-t)}\right] d t+ \\
+\int_{y}^{\beta} g(t)\left[A_{k}(y) e^{-\lambda_{k} t}-B_{k}(y) e^{-\lambda_{k}(\beta-t)}-e^{-\lambda_{k}(t-y)}\right] d t= \\
=\int_{0}^{y} g(t) E_{k}(t, y) d t+\int_{y}^{\beta} g(t) H_{k}(t, y) d t .
\end{aligned}
$$

Здесь

$$
\begin{aligned}
& E_{k}(t, y)=A_{k}(y) e^{-\lambda_{k} t}-B_{k}(y) e^{-\lambda_{k}(\beta-t)}-e^{-\lambda_{k}(y-t)}, \\
& H_{k}(t, y)=A_{k}(y) e^{-\lambda_{k} t}-B_{k}(y) e^{-\lambda_{k}(\beta-t)}-e^{-\lambda_{k}(t-y)}
\end{aligned}
$$

Тогда с учетом (72) и (74) равенство (73) принимает вид

$$
\begin{gathered}
\sum_{k=1}^{\infty} \frac{f_{k}}{2 \lambda_{k}}\left(\int_{0}^{y} g(t) E_{k}(t, y) d t+\int_{y}^{\beta} g(t) H_{k}(t, y) d t\right) \sin \mu_{k} x_{0}= \\
=d(y)-\sum_{k=1}^{\infty} C_{k}(y) \sin \mu_{k} x_{0}=\tilde{d}(y)
\end{gathered}
$$

В силу теоремы 3 ряд (22) сходится равномерно на замкнутой области $\bar{D}$, поэтому в левой части равенства (75), переставляя операции суммирования и интегрирования между собой, получим интегральное уравнение типа первого рода:

$$
\int_{0}^{y} g(t) E(t, y) d t+\int_{y}^{\beta} g(t) H(t, y) d t=\widetilde{d}(y)
$$

где

$$
\begin{aligned}
& E(t, y)=\sum_{k=1}^{\infty} \frac{f_{k}}{2 \lambda_{k}} E_{k}(t, y) \sin \mu_{k} x_{0}, \\
& H(t, y)=\sum_{k=1}^{\infty} \frac{f_{k}}{2 \lambda_{k}} H_{k}(t, y) \sin \mu_{k} x_{0} .
\end{aligned}
$$

При этом заметим, что

$$
\widetilde{d}(0)=d(0)-\sum_{k=1}^{\infty} C_{1 k}(0) \sin \mu_{k} x_{0}=d(0)-\varphi\left(x_{0}\right)=0
$$


и левая часть уравнения (76) при $y=0$ равна нулю.

Обе части уравнения (76) продифференцируем. В результате получим

$$
g(y)[E(y, y)-H(y, y)]+\int_{0}^{y} g(t) \frac{\partial E(t, y)}{\partial y} d t+\int_{y}^{\beta} g(t) \frac{\partial H(t, y)}{\partial y} d t=\widetilde{d}^{\prime}(y)
$$

При этом из соотношений (77) и (78) следует, что

$$
E(y, y)-H(y, y)=\sum_{k=1}^{\infty} \frac{f_{k}}{2 \lambda_{k}}\left[E_{k}(y, y)-H_{k}(y, y)\right] \sin \mu_{k} x_{0} \equiv 0
$$

так как

$$
E_{k}(y, y)-H_{k}(y, y) \equiv 0 \text {. }
$$

Тогда, еще раз дифференцируя уравнение (79), будем иметь

$$
\begin{aligned}
g(y)\left[\frac{\partial E(t, y)}{\partial y}-\frac{\partial H(t, y)}{\partial y}\right]_{t=y} & +\int_{0}^{y} g(t) \frac{\partial^{2} E(t, y)}{\partial y^{2}} d t+ \\
& +\int_{y}^{\beta} g(t) \frac{\partial^{2} H(t, y)}{\partial y^{2}} d t=\widetilde{d}^{\prime \prime}(y) .
\end{aligned}
$$

Теперь на основании (77) и (78) вычислим

$$
\begin{array}{r}
{\left[\frac{\partial E(t, y)}{\partial y}-\frac{\partial H(t, y)}{\partial y}\right]_{t=y}=\sum_{k=1}^{\infty} \frac{f_{k}}{2 \lambda_{k}}\left[\frac{\partial E_{k}(t, y)}{\partial y}-\frac{\partial H_{k}(t, y)}{\partial y}\right] \sin \mu_{k} x_{0}=} \\
=\sum_{k=1}^{\infty} f_{k} \sin \mu_{k} x_{0}=f\left(x_{0}\right)
\end{array}
$$

Если потребовать что $f\left(x_{0}\right) \neq 0$, то из $(80)$ и $(81)$ получим интегральное уравнение Фредгольма второго рода:

$$
g(y)-\lambda \int_{0}^{\beta} g(t) K(t, y) d t=\mu(y)
$$

где

$$
\begin{gathered}
K(t, y)= \begin{cases}\frac{\partial^{2} E(t, y)}{\partial y^{2}}, & 0 \leqslant t \leqslant y \\
\frac{\partial^{2} H(t, y)}{\partial y^{2}}, & y \leqslant t \leqslant \beta\end{cases} \\
\frac{\partial^{2} E(t, y)}{\partial y^{2}}=\sum_{k=1}^{\infty} \frac{f_{k}}{2 \lambda_{k}} \frac{\partial^{2} E_{k}(t, y)}{\partial y^{2}} \sin \mu_{k} x_{0}=\sum_{k=1}^{\infty} \frac{\lambda_{k}}{2} f_{k} E_{k}(t, y) \sin \mu_{k} x_{0} \\
\frac{\partial^{2} H(t, y)}{\partial y^{2}}=\sum_{k=1}^{\infty} \frac{f_{k}}{2 \lambda_{k}} \frac{\partial^{2} H_{k}(t, y)}{\partial y^{2}} \sin \mu_{k} x_{0}=\sum_{k=1}^{\infty} \frac{\lambda_{k}}{2} f_{k} H_{k}(t, y) \sin \mu_{k} x_{0}
\end{gathered}
$$




$$
\lambda=-1 / f\left(x_{0}\right), \quad \mu(y)=\tilde{d}^{\prime \prime}(y) / f\left(x_{0}\right) .
$$

Заметим, что функции $E_{k}(t, y)$ и $H_{k}(t, y)$ имеют производные любого порядка соответственно на замкнутых областях $0 \leqslant t \leqslant y \leqslant \beta$ и $0 \leqslant y \leqslant t \leqslant \beta$, и $E_{k}(y, y)=H_{k}(y, y)$, а по переменной $k$ ограничены. Тогда равномерная сходимость рядов (84) и $(85)$ зависит от $f_{k}$, т.е. от гладкости функции $f(x)$ на $[0, l]$. Если функция $f(x) \in C^{2}[0, l]$ и $f(0)=f(l)=0$, то

$$
f_{k}=-\frac{1}{\mu_{k}^{2}} \frac{2}{l} \int_{0}^{l} f^{\prime \prime}(x) \sin \mu_{k} x d x=-\frac{f_{k}^{(2)}}{\mu_{k}^{2}}
$$

и ряды (84) и (85) будут равномерно сходиться на указанных областях. Поэтому ядро $K(t, y)$, определенное формулой $(83)$, непрерывно в замкнутом квадрате $0 \leqslant t, y \leqslant \beta$. Если $d(y) \in C^{2}[0, \beta]$ и функции $\varphi(x)$ и $\widetilde{\psi}(x)$ удовлетворяют условиям теоремы 3 , то функция $\mu(y)$ непрерывна на $[0, \beta]$. Итак, уравнение (82) представляет собой интегральное уравнение Фредгольма второго рода с непрерывным ядром и непрерывной правой частью. Тогда методом последовательных приближений можно доказать однозначную разрешимость данного уравнения в классе непрерывных на $[0, \beta]$ функций при $|\lambda|<1 /(M l)$, где $M=\max _{\bar{D}}|K(t, y)|$. Из теории Фредгольма следует также, что если $\lambda$ не является характеристическим числом ядра $K(t, y)$, то интегральное уравнение (82) имеет непрерывное на $[0, \beta]$ решение.

Таким образом, нами доказана следующая

Teоpema 11. Пусть $\varphi(x) \in C^{3}[0, l], \varphi(0)=\varphi^{\prime \prime}(0)=\varphi(l)=\varphi^{\prime \prime}(l)=0$, $\widetilde{\psi}(x) \in C^{2}[0, l], \widetilde{\psi}(0)=\widetilde{\psi}(l)=0, d(y) \in C^{2}[0, \beta], d(0)=\varphi\left(x_{0}\right), f(x) \in C^{2}[0, l]$, $f(0)=f(l)=0, f\left(x_{0}\right) \neq 0$. Тогда единственное решение задачи 6 существует при выполнении одного из следующих условий:

1) $\left|f\left(x_{0}\right)\right|>M l$,

2) число $-f^{-1}\left(x_{0}\right)$ не является характеристическим числом ядра $K(t, y)$. При этом функиия $g(y)$ определяется как решение интегрального уравнения (82) в классе $C[0, \beta]$, после чего функиия $и(x, y)$ находится как решение задачи 2 по бормуле (22) и принадлежит $C^{2}(\bar{D})$.

Отметим, что в теореме 11 условие $f\left(x_{0}\right) \neq 0$ существенно, здесь $\widetilde{x}_{0}=$ $=x_{0} / l \in(0,1)$. Пусть при некоторых $\widetilde{x}_{0}$ и $n=p \in \mathbb{N}$ выражение $\sin \pi k \widetilde{x}_{0}=0$. Тогда для функций $f(x)=\sin \mu_{p} x$ и $g(y) \in C[0, \beta]$ аналогично (63)-(65) строится ненулевое решение однородной задачи 6 (где $u(0, y)=u(l, y) \equiv 0$, $\varphi(x)=\widetilde{\psi}(x) \equiv 0, d(y) \equiv 0):$

$$
u(x, y)=\widetilde{u}_{p}(y) \sin \mu_{p} x,
$$

где

$$
\widetilde{u}_{p}(y)=\frac{f_{p}}{2 \lambda_{p}}\left[g_{1 p}(0) A_{p}(y)-g_{2 p}(\beta) B_{p}(y)-g_{1 p}(y)-g_{2 p}(y)\right],
$$

$f_{p} \neq 0$ - произвольная постоянная. Заметим, что здесь $f\left(x_{0}\right)=0$.

Следовательно, в силу построенного примера (86), однозначная разрешимость задачи 6 зависит также от выбора точки $\widetilde{x}_{0}$, т.е. от отношения $x_{0} / l$. Соотношение $\sin \pi k \widetilde{x}_{0}=0 \Leftrightarrow \widetilde{x}_{0}=p / q$, где $p, q \in \mathbb{N}$, т.е. тогда, когда отношение $x_{0} / l$ является рациональным числом из интервала $(0,1)$. 
В заключение отметим, что аналогично можно исследовать аналоги задачи 6 на основании граничных условий $\left(B_{2}\right)-\left(B_{4}\right)$. В этих случаях относительно неизвестной функции $g(y)$ получится интегральное уравнение типа $(82)$.

Конкурирующие интересы. Мы не имеем конкурирующих интересов.

Авторский вклад и ответственность. Все авторы принимали участие в разработке концепции статьи и в написании рукописи. Авторы несут полную ответственность за предоставление окончательной рукописи в печать. Окончательная версия рукописи была одобрена всеми авторами.

Финансирование. Работа выполнена при финансовой поддержке Российского фонда фундаментальных исследований (проект № 16-31-00421_мол_а), Российского фонда фундаментальных исследований и Республики Башкортостан (проект № 1741-020516_p_a).

\section{Библиографический список}

1. Сабитов К. Б., Мартемьянова Н. В. Нелокальная обратная задача для уравнения смешанного типа // Изв. вузов. Матем., 2011. № 2. С. 71-85.

2. Сабитов К. Б., Хаджи И. А. Краевая задача для уравнения Лаврентьева-Бицадзе с неизвестной правой частью // Изв. вузов. Матем., 2011. № 5. С. 44-52.

3. Сабитов К. Б., Мартемьянова Н. В. Обратная задача для уравнения эллиптикогиперболического типа с нелокальным граничным условием// Сиб. матем. журн., 2012. T. 53, № 3. С. 633-647.

4. Сабитов К. Б., Мартемьянова Н. В. Обратная задача для уравнения ЛаврентьеваБицадзе, связанная с поиском элементов правой части // Изв. вузов. Матем., 2017. № 2. C. $44-57$.

5. Сабитов К. Б., Мартемьянова Н. В. Нелокальная обратная задача для уравнения с оператором Лавретьева-Бицадзе в прямоугольной области// Доклады AMAH, 2013. T. 15, № 2. C. 73-86.

6. Sabitov K. B., Martemyanova N. V. Nonlocal boundary value problem for the third order equation of mixed type// Contemporary Analysis and Applied Mathematics, 2015. vol.3, no. 2. pp. 153-169. doi: 10.18532/ caam. 52633.

7. Крайко А. Н., Макаров В. Е., Пудовиков Д. Е. К построению головной ударной волны при "обратном" расчете сверхзвукового течения методом характеристик // ЖК. вычисл. матем. и матем. физ., 1999. Т. 39, №11. С. 1889-1894.

8. Крайко А. Н., Пьянков К. С. Построение профилей и мотогондол, суперкритических в околозвуковом потоке идеального газа // Ж. вычисл. матем. и матем. физ., 2000. T. 40, № 12. C. 1890-1904.

9. Лавретьев М. М., Романов В. Г., Шишатский С. П. Некорректные задачи математической физики и анализа. М.: Наука, 1980. 287 с.

10. Лавретьев М. М., Резницкая К. Г., Яхно В. Г. Одномерные обратные задачи математической физики. Новосибирск: Наука, 1982. 88 с.

11. Романов В. Г. Обратнъе задачи математической физики. М.: Наука, 1984. 264 с.

12. Романов В. Г., Кабанихин С. И. Обратные задачи геоэлектрики. М.: Наука, 1991. 304 с.

13. Денисов А. М. Введение в теорию обратных задач. М.: МГУ, 1994. 206 с.

14. Prilepko A. I., Orlovsky D. G., Vasin I. A. Methods for Solving Inverse Problems in Mathematical Physics / Monographs and Textbooks in Pure and Applied Mathematics. vol. 231. Boca Raton: CRC Press, 2000. xiv+709 pp. doi : 10.1201/9781482292985

15. Isakov V. Inverse problems for partial differential equations / Applied Mathematical Sciences. vol. 127. New York: Springer, 2006. xiii+346 pp. doi : 10.1007/0-387-32183-7

16. Кабанихин С. И. Обратные и некорректные задачи. Новосибирск: Сибирское научное изд-во, 2009. 
17. Соловьев В. В. Обратные задачи определения источника для уравнения Пуассона на плоскости // Ж. вычисл. матем. и матем. физ., 2004. Т. 44, № 5. С. 862-871.

18. Соловьев В. В. Обратные задачи для эллиптических уравнений на плоскости. I // Дифферени. уравнения, 2006. Т. 42, № 8. С. 1106-1114.

19. Соловьев В. В. Обратные задачи для эллиптических уравнений на плоскости. II // Дифферени. уравнения, 2007. Т. 43, №1. С. 101-109.

20. Соловьев В. В. Обратные задачи для уравнений эллиптического и параболического типов в пространствах Гельдера: Автореферат диссерт. ... д-ра физ.-мат. наук.. М., 2014.

21. Орловский Д. Г. Об одной обратной задаче для дифференциального уравнения второго порядка в банаховом пространстве // Дифферени. уравнения, 1989. Т. 25, №6. С. 10001009.

22. Орловский Д. Г. К задаче определения параметра эволюционного уравнения // Дифферени. уравнения, 1990. Т. 26, № 9. С. 1614-1621.

23. Орловский Д. Г. Обратная задача Дирихле для уравнения эллиптического типа // Дифферени. уравнения, 2008. Т. 44, № 1. С. 119-128.

24. Сабитов К. Б. Уравнения математической физики. М.: Физматлит, 2013. 
MSC: 35R30, 35M13

\title{
On the question of the correctness of inverse problems for the inhomogeneous Helmholtz equation
}

\author{
K. B. Sabitov ${ }^{1,2}$, N. V. Martem'yanova ${ }^{3}$ \\ 1 Samara State Technical University, \\ 244, Molodogvardeyskaya st., Samara, 443100, Russian Federation. \\ 2 Samara State University of Social Sciences and Education, \\ 65/67, M. Gorkiy str., Samara, 443099, Russian Federation. \\ 3 Samara National Research University, \\ 34, Moskovskoye shosse, Samara, 443086, Russian Federation.
}

\begin{abstract}
In the rectangular domain, the initial-boundary value problem for the Helmholtz equation and its non-local modifications are studied and the inverse problems for finding its right-hand side are studied. The solutions of direct problems with nonlocal boundary conditions and inverse problems are constructed in explicit form as the sums of orthogonal series in the system of eigenfunctions of the one-dimensional Sturm-Liouville spectral problem. The corresponding uniqueness theorems for the solution of all set problems are proved. Sufficient conditions for boundary functions are established, which are guaranteed by the existence and stability theorems for the solution of the proposed new problem statements.
\end{abstract}

Keywords: Helmholtz equation, initial-boundary value problem, nonlocal problems, inverse problems, uniqueness, existence, series, stability, integral equations.

Received: $10^{\text {th }}$ January, 2018 / Revised: 21 ${ }^{\text {st }}$ April, $2018 /$

Accepted: $11^{\text {th }}$ June, $2018 /$ First online: $27^{\text {th }}$ June, 2018

Competing interests. We have no competing interests.

Authors' contributions and responsibilities. Each author has participated in the article concept development and in the manuscript writing. The authors are absolutely

\section{Research Article}

(우(;) The content is published under the terms of the Creative Commons Attribution 4.0 International License (http://creativecommons.org/licenses/by/4.0/)

Please cite this article in press as:

Sabitov K. B., Martem'yanova N. V. On the question of the correctness of inverse problems for the inhomogeneous Helmholtz equation, Vestn. Samar. Gos. Tekhn. Univ., Ser. Fiz.-Mat. Nauki [J. Samara State Tech. Univ., Ser. Phys. Math. Sci.], 2018, vol. 22, no. 2, pp. 269-292. doi: 10.14498/vsgtu1600 (In Russian).

\section{Authors' Details:}

Kamil B. Sabitov (1) http://orcid.org/0000-0001-9516-2704

Dr. Phys \& Math. Sci.; Professor, Dept. of Higher Mathematics ${ }^{1}$, Professor, Dept. of Physics, Mathematics and Methods of Teaching ${ }^{2}$; e-mail: sabitov_fmf@mail.ru

Nina V. Martem'yanova (10) http://orcid.org/0000-0001-8025-0234

Cand. Phys \& Math. Sci.; Associate Professor; Dept. of Higher Mathematics ${ }^{3}$;

e-mail: ninamartem@yandex.ru 
responsible for submitting the final manuscript in print. Each author has approved the final version of manuscript.

Funding. This paper was written with the support of the Russian Foundation for Basic Research (grant no. 16-31-00421_mol_a), Russian Foundation for Basic Research and Republic Bashkortostan (grant no. 17-41-020516_r_a).

\section{References}

1. Sabitov K. B., Martem'yanova N. V. A nonlocal inverse problem for a mixed-type equation, Russian Math. (Iz. VUZ), 2011, vol.55, no. 2, pp. 61-74. doi: 10.3103/S1066369X11020083.

2. Sabitov K. B., Khadzhi I. A. The boundary-value problem for the Lavrent'ev-Bitsadze equation with unknown right-hand side, Russian Math. (Iz. VUZ), 2011, vol. 55, no. 5, pp. 35-42. doi : 10.3103/S1066369X11050069.

3. Sabitov K. B., Martem'yanova N. V. An inverse problem for an equation of elliptichyperbolic type with a nonlocal boundary condition, Siberian Math. J., 2012, vol. 53, no. 3, pp. 507-519. doi : 10.1134/S0037446612020310.

4. Sabitov K. B., Martem'yanova N. V. The inverse problem for the Lavrent'ev-Bitsadze equation connected with the search of elements in the right-hand side, Russian Math. (Iz. VUZ), 2017, vol. 61, no. 2, pp. 36-48. doi: 10.3103/S1066369X17020050.

5. Sabitov K. B., Martem'yanova N. V. Nonlocal inverse problem for the equation with the Lavret'ev-Bitsadze operator in a rectangular domain, Doklady AMAN, 2013, vol. 15, no. 2, pp. 73-86 (In Russian).

6. Sabitov K. B., Martem'yanova N. V. Nonlocal boundary value problem for the third order equation of mixed type, Contemporary Analysis and Applied Mathematics, 2015, vol. 3, no. 2, pp. 153-169. doi: 10.18532/caam.52633.

7. Krajko A. N., Makarov V. E., Pudovikov D. E. Construction of the bow wave through upstream computation of a supersonic flow by the method of characteristics, Comput. Math. Math. Phys., 1999, vol. 39, no. 11, pp. 1814-1819.

8. Krajko A. N., P'yankov K. S. Construction of airfoils and engine nacelles that are supercritical in a transonic perfect-gas flow, Comput. Math. Math. Phys., 2000, vol.40, no. 12, pp. $1816-1829$.

9. Lavrent'ev M. M., Romanov V. G., Shishatskii S. P. Nekorrektnye zadachi matematicheskoy fiziki $i$ analiza [Ill-posed Problems of Mathematical Physics and Analysis]. Moscow, Nauka, 1980, 287 pp. (In Russian)

10. Lavrent'ev M. M., Reznitskaya K. G., Yakhno V. G. Odnomernye obratnye zadachi matematicheskoy fiziki [One-dimensional Inverse Problems of Mathematical Physics]. Novosibirsk, Nauka, 1982, 88 pp. (In Russian)

11. Romanov V. G. Obratnye zadachi matematicheskoy fiziki [Inverse Problems of Mathematical Physics]. Moscow, Nauka, 1984, 264 pp. (In Russian)

12. Romanov V. G., Kabanikhin S. I. Obratnye zadachi geoelektriki [Inverse Problems of Geoelectrics]. Moscow, Nauka, 1991, 304 pp. (In Russian)

13. Denisov A. M. Elements of the Theory of Inverse Problems, Inverse and Ill-Posed Problems Series, vol. 14. Utrecht, VSP Press, 1999, viii +272 pp. doi: 10.1515/9783110943252

14. Prilepko A. I., Orlovsky D. G., Vasin I. A. Methods for Solving Inverse Problems in Mathematical Physics, Monographs and Textbooks in Pure and Applied Mathematics, vol. 231. Boca Raton, CRC Press, 2000, xiv+709 pp. doi : 10.1201/9781482292985

15. Isakov V. Inverse problems for partial differential equations, Applied Mathematical Sciences, vol. 127. New York, Springer, 2006, xiii+346 pp. doi: 10.1007/0-387-32183-7

16. Kabanikhin S. I. Inverse and Ill-posed Problems, Inverse and Ill-posed Problems. Theory and Applications, vol.55. Utrecht, VSP Press, 2011, xvi+459 pp. doi: 10.1515/9783110224016

17. Solov'ev V. V. Inverse problems of source determination for the Poisson equation on the plane, Comput. Math. Math. Phys., 2004, vol.44, no. 5, pp. 815-824. 
18. Solov'ev V. V. Inverse problems for elliptic equations on the plane: I, Differ. Equ., 2006, vol. 42, no. 8, pp. 1170-1179. doi: 10.1134/S0012266106080118.

19. Solov'ev V. V. Inverse problems for elliptic equations on the plane: II, Differ. Equ., 2007, vol. 43, no. 1, pp. 108-117. doi: 10.1134/s0012266107010119.

20. Solov'ev V. V. Inverse problems for equations of elliptic and parabolic type in Hölder spaces, Thesis, Doctor of Phys.-Math. Sci. Moscow, 2014.

21. Orlovsky D. G. On an inverse problem for a second order differential equation in Banach space, Differ. Equ., 1989, vol. 25, no. 6, pp. 730-738.

22. Orlovsky D. G. Determination of a parameter of an evolution equation, Differ. Equ., 1990, vol. 26, no. 9, pp. 1201-1207.

23. Orlovsky D. G. Inverse Dirichlet problem for an equation of elliptic type, Differ. Equ., 2008, vol. 44, no. 1, pp. 124-134.

24. Sabitov K. B. Uravneniia matematicheskoy fiziki [Equations of mathematical physics]. Moscow, Fizmatlit, 2013 (In Russian). 